\title{
Inhibition of PTEN Activity Aggravates Post Renal Fibrosis in Mice with Ischemia Reperfusion-Induced Acute Kidney Injury
}

\author{
Jun Zhou Jiying Zhong ${ }^{a}$ Sen Lin ${ }^{a}$ Zhenxing Huang ${ }^{a}$ Hongtao Chen ${ }^{b}$ \\ Simin Tang ${ }^{\mathrm{a}}$ Chengxiang Yang ${ }^{\mathrm{a}}$ Youling Fanc \\ a Department of Anesthesiology, The First People's Hospital of Foshan, Foshan, Guangdong Province, \\ ${ }^{b}$ Department of Anesthesiology, Eighth People's Hospital of Guangzhou, Guangzhou, Guangdong \\ Province, ' Department of Anesthesiology, Panyu Central Hospital, Guangzhou, Guangdong Province, \\ China
}

\section{Key Words}

Chronic kidney disease $\cdot$ Fibrosis $•$ Cytokines $•$ Fibroblast $•$ PTEN

\begin{abstract}
Background: Renal fibrosis is a common pathophysiological feature of chronic kidney disease. Acute kidney injury (AKI) is defined as an independent causal factor of chronic kidney disease, with a pathological representation of post renal fibrosis. However, the etiopathogenesis underlying post renal fibrosis induced by AKI is not completely understood. Methods: BALB/C mice were treated with bpv or vehicle controls and were, respectively, the ischemia reperfusion (IR) model group and control group. All of the animals had blood taken from the orbital venous plexus at 24 hours after IR. Six mice in each group were randomly chosen and euthanized 7 days after IR treatment, and the remaining six mice in each group were euthanized 14 days after IR treatment. We examined the effect on post kidney fibrosis of inhibiting PTEN activity in mice in an IR induced AKI experimental model. Results: Compared with vehicle mice, bpv(PTEN specific inhibitor) treated mice accumulated more bone marrow-derived fibroblasts and myofibroblasts in the kidneys. Inhibition of PTEN activity increased the expression of $\alpha$-smooth muscle actin and extracellular matrix proteins and post kidney fibrosis. Furthermore, inhibition of PTEN activity resulted in more inflammatory cytokines in the kidneys of mice subjected to IR-induced renal fibrosis. Moreover, inhibition of PTEN activity up-regulated PI3K protein expression and Akt phosphorylation. Conclusions: Our study demonstrated that PTEN played an important role in post renal fibrosis in mice with ischemia reperfusion-induced AKI. These results indicated that the PTEN/PI3K/Akt signaling pathway may serve as a novel therapeutic target for AKI-induced chronic kidney disease.
\end{abstract}




\section{Introduction}

Acute kidney injury (AKI) is an increasingly common complication of hospital admission and the leading clinical cause of chronic kidney disease (CKD), with high morbidity and mortality $[1,2]$. The transition of AKI to CKD is of major clinical significance. Preventing and treating AKI-induced CKD is a serious global health challenge. Fibrosis in response to injury is often perceived to be a pathologic hallmark of $\operatorname{CKD}[3,4]$. A large amount of extracellular matrix (ECM) produced by activated fibroblasts gives rise to loss of useful renal parenchyma and kidney function [5]. Despite more and more attention to the diverse aspects related to AKI-induced CKD, the process from acute injury to renal fibrosis remains unclear. Therefore, understanding the mechanism of renal fibrosis induced by AKI is essential for developing novel therapeutic strategies and may have important clinical implications.

Phosphatase and tensin homolog deleted on chromosome ten (PTEN) was previously regarded as a general regulatory factor in the evolution of various solid tumors [6]. Loss of PTEN function, one of the most common driving events in cancer development [7], resulted in dysregulated activation of the phosphoinositide 3-kinase (PI3K) signaling network. Recently, more scholars have discovered that PTEN plays an important role in mediating fibrosis development in vital organs such as the liver, lung and kidneys by activating profibrotic signaling pathways [8-10]. However, the molecular mechanisms of the PTEN pathway underlying renal fibrosis induced by AKI are unclear. In addition to the role of interstitial fibroblast proliferation, there were studies indicating that bone marrow-derived fibroblast precursors contributed significantly to the development of renal fibrosis $[11,12]$. Previous research demonstrated that activation of proinflammatory cytokines contributed substantially to the development of renal interstitial fibrosis [13, 14]. Moreover, mounting evidence in vivo and in vitro has indicated that PTEN mediates multiple signal pathways involved in the pathophysiologic process of fibrosis in the kidney such as SMAD3, p53, and JNK[15, 16]. Here, we investigated the inhibition of PTEN activity by bisperoxovanadium $(\mathrm{bpV})$ in post renal fibrosis induced by AKI with ischemia reperfusion (IR) models in mice. $\mathrm{BpV}$ compounds are dual protein-lipid phosphatases best known for their potent and specific inhibition of PTEN, which usually has been characterized using in vitro phosphatase assays and Western blot analyses of the phosphorylation of Akt, a downstream target of the PTEN pathway [17]. Our results demonstrated that inhibition of PTEN activity exacerbated the development of post renal fibrosis induced by AKI. The possible molecular mechanism indicated that PTEN mediates the signaling transduction of PI3K/Akt, which led to inflammatory cytokine migration, interstitial fibroblast proliferation and myeloid fibroblast activation.

\section{Materials and Methods}

\section{Animals}

The animal experiments were conducted according to the guidelines of laboratory animal care and were approved by the Institutional Animal Care and Use Committee of the First People hospital of Foshan. BALB/c mice with bpv treatment and vehicle controls served as IR models and sham controls, respectively. The implementation of IR-induced AKI model steps are described below. Forty-eight mice, 8 to 12 weeks old, twelve in each group, were anesthetized by intraperitoneal injection of ketamine $(80 \mathrm{mg} / \mathrm{kg})$ and xylazine $(10 \mathrm{mg} / \mathrm{kg})$. The kidneys were exposed through a flank incision and were subjected to ischemia by clamping the renal pedicles with non-traumatic microaneurysm clamps. After 30 minutes, the clamps were removed, and blood reflow was confirmed. Body temperature was maintained at $36.5-37.5^{\circ} \mathrm{C}$ throughout the procedure. Sham control mice underwent an identical surgical procedure but without pedicle clamping. All of the animals had blood sampled $(0.3 \mathrm{ml})$ from the orbital venous plexus at 24 hours after reperfusion with a capillary tube. Six mice in each group were randomly chosen and euthanized 7 days after IR treatment, and the remaining six mice in each group were euthanized 14 days after IR treatment. Kidneys were perfused and harvested. 


\section{Cellular Physiology Cell Physiol Biochem 2017;43:1841-1854 \\ \begin{tabular}{l|l|l} 
and Biochemistry $10.1159 / 000484070$ & $\begin{array}{l}\text { C) } 2017 \text { The Author(s). Published by S. Karger AG, Basel } \\
\text { www.karger.com/cpb }\end{array}$ \\
\hline
\end{tabular}}

Zhou et al.: Molecular Mechanisms of Post Renal Fibrosis Induced by Acute Kidney Injury (AKI)

\section{Measurement of Renal Function}

Blood creatinine was measured using a creatinine assay kit (BioAssay Systems, Hayward, CA) according to the manufacturer's instructions. Blood urea nitrogen was determined fluorometrically as previously described [18].

\section{PTEN activity}

The PTEN Activity ELISA is designed to quantify the phosphatase activity of PTEN by detection of the product, PIP2, in a competitive ELISA format, eliminating the need for radioactivity, organic solvents, and thin layer chromatography. PTEN activity was detected according to the assay kit instructions (K-4700-1kit, Echelon, USA).

\section{Renal Morphology}

Mice were perfused with PBS to remove blood. A portion of the kidney tissue was embedded in paraffin and cut to $4-\mu \mathrm{m}$ thickness. After deparaffinization and rehydration, sections were stained with Sirius red to evaluate collagen fibers. The Sirius red-stained sections were scanned with a microscope equipped with a digital camera (Nikon Instruments), and quantitative evaluation was performed using NIS-Elements $\mathrm{Br}$ 3.0 software as described [19]. The Sirius red-stained area was calculated as a percentage of the total area.

\section{Immunofluorescence}

Fibronectin, Collagen I, and $\alpha$-SMA staining was performed on paraffin sections. After fixation and antigen retrieval, non-specific binding was blocked using a protein block (Dako). Kidney sections were then incubated with primary antibody followed by Alexa-488 conjugated secondary antibody. Slides were mounted with medium containing DAPI. The fluorescence intensity was visualized using a microscope equipped with a digital camera (Nikon Instruments). The fluorescence positive area was calculated as a percentage of the total area, as previously described [12]. For double immunofluorescence, kidney tissues were embedded in OCT compound, cut to $5-\mu \mathrm{m}$ thickness, and mounted on Superfrost Plus slides. Kidney sections were fixed and stained with platelet-derived growth factor receptor $\beta$ (PDGFRR- $\beta$ ) (Santa Cruz Biotechnology) and rat anti-CD45 (BD Biosciences) or anti-CD206 (Bio-Rad Laboratories), followed by appropriate secondary antibodies. Slides were mounted with medium containing DAPI. Confocal images were obtained using a Zeiss LSM 780 confocal microscope.

\section{Western Blot Analysis}

Protein was extracted using RIPA buffer containing cocktail proteinase inhibitors and quantified with a Bio-Rad protein assay. An equal amount of protein was separated on SDS-polyacrylamide gels in a Tris/SDS buffer system and then transferred onto nitrocellulose membranes. The membranes were incubated with primary antibodies overnight, followed by incubation with appropriate fluorescence-conjugated secondary antibodies. The proteins of interest were analyzed using an Odyssey IR scanner (LI-COR Biosciences), and signal intensities were quantified using NIH Image/J software (National Institutes of Health).

\section{Immunohistochemistry}

Immunohistochemical staining was performed on paraffin sections. Antigen retrieval was performed with an antigen unmasking solution (Vector Laboratories) or proteinase K. The endogenous peroxidase activity was quenched with $3 \% \mathrm{H}_{2} \mathrm{O}_{2}$ for $10 \mathrm{~min}$. After blocking with $5 \%$ normal serum, slides were incubated with primary antibodies in a humidified chamber overnight. After washing, slides were incubated with appropriate secondary antibodies and ABC solution sequentially according to the ABC kit (Vector Laboratories). Slides were then visualized by incubation in diaminobenzidine solution for an appropriate duration. Nuclear staining was performed with hematoxylin. The slides were dehydrated, cleared, and mounted. The images from these slides were obtained and analyzed by NIS Element software (Nikon Instruments) using the Nikon microscope image system (Nikon Instruments).

\section{Quantitative Real-Time RT-PCR}

Total RNA was extracted from kidney tissues with TRIzol reagent (Invitrogen). Aliquots $(1 \mu \mathrm{g})$ of total RNA were reverse transcribed using Super-Script II reverse transcriptase. Real-time PCR was performed using IQ SYBR green super-mix reagent (Bio-Rad, Hercules, CA) with a Bio-Rad real-time PCR machine 

Injury (AKI)

according to the manufacturer's instructions. The comparative Ct method ( $\Delta \Delta \mathrm{Ct}$ ) was used to quantify gene expression, and the relative quantification was calculated as $2^{-\Delta \Delta C t}$. The expression level of targeted genes were normalized to the GAPDH level for each sample. The primer sequences were:

IL-1 $\beta$-forward, 5' CTCGGCCAAGACAGGTCGCTC 3'

-reverse, 5' CCCCCACACGTTGACAGCTAGG 3'

IL-6-forward, 5' AGGATACCACTCCCAACAGACCTG-3',

-reverse, 5' CTGCAAGTGCATCATCGTTGTTCA-3';

TNF- $\alpha$-forward, 5' CATGAGCACAGAAAGCATGATCCG-3',

-reverse, 5' AAGCAGGAATGAGAAGAGGCTGAG-3';

NF- $\kappa$ B-forward, 5' GAGACATCCTTCCGCAAACT 3'

-reverse, $5^{\prime}$ 'TCCTTCCTGCCCATAATCA 3'

GAPDH-forward, 5' CCAATGTGTCCGTCGCGTGGATCT-3',

-reverse, 5' GTTGAAGTCGCAGGAGACAACC-3'.

\section{Statistical Analysis}

The data were presented as the means \pm SEM. Multiple groups of comparisons were performed by ANOVA followed by the Bonferroni procedure, A P value $<0.05$ was considered statistically significant.

\section{Results}

\section{The effect of bpv on PTEN activity}

BALB/c mice were subjected to i.p. injection of a sham preparation or bpv at $200 \mu \mathrm{g} / \mathrm{kg}$ once every $4 \mathrm{~h}$. We determined the activity of PTEN in the kidneys of mice after IR and the effect of PTEN activity in the kidneys of bpv-treated mice 7 days after IR. Using an enzymelinked immuno sorbent assay (ELISA), we found that the activity of PTEN was up-regulated significantly in the kidneys of IR-treated mice compared with sham controls. PTEN activity was inhibited significantly in the kidneys of bpv-treated mice compared with vehicle controls (Fig. 1A).

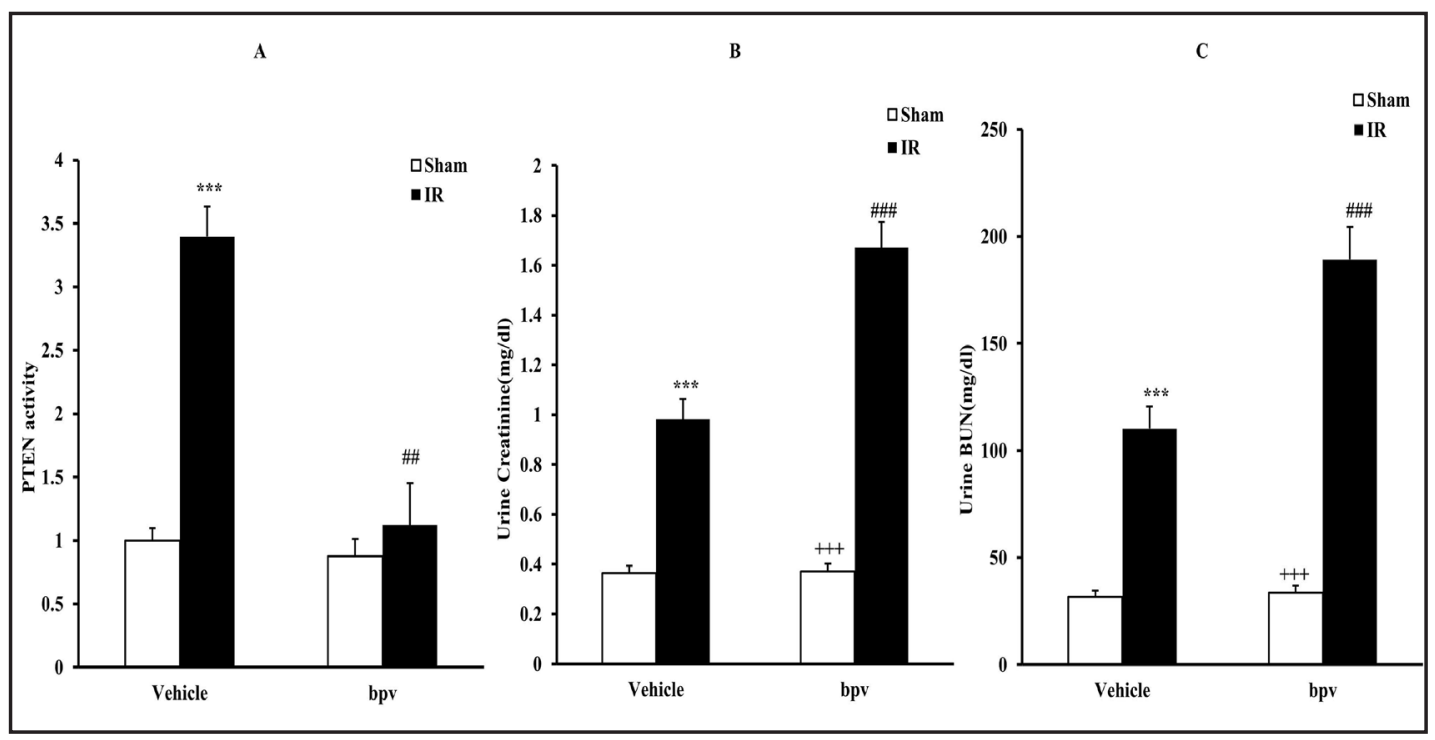

Fig. 1. PTEN activity in renal tissue and kidney function in IR-treated mice.A. PTEN activity by ELISA in renal tissue of vehicle and bpv mice after sham or IR treatment for 7 days. ${ }^{* * *} p<0.001$ vs. Vehicle Sham; $\#$ \# $<0.01$ vs. Vehicle IR, $n=6$ each. B. Effect on serum creatinine in vehicle and bpv mice at $24 \mathrm{~h}$ after IR or sham treatment. ${ }^{* * *} \mathrm{p}<0.001$ vs. Vehicle Sham; ${ }^{+++} \mathrm{p}<0.001$ vs. bpv IR; ${ }^{\# \#} \mathrm{p}<0.001$ vs. Vehicle IR, $\mathrm{n}=12$ each. C. Effects on serum urea nitrogen in vehicle and bpv mice at $24 \mathrm{~h}$ after cisplatin or sham treatment. ${ }^{* * *} \mathrm{p}<0.001$ vs. Vehicle Sham; ${ }^{+++} \mathrm{p}<0.001$ vs. bpv IR; ${ }^{\# \# ~} \mathrm{p}<0.001$ vs. Vehicle IR, $\mathrm{n}=12$ each.

\section{KARGER}



Injury (AKI)

Verifying the reliability of the IR model with detection of renal function

To verify the reliability of the IR model, we detected renal function with serum creatinine and urea nitrogen $24 \mathrm{~h}$ after IR treatment. Mice developed renal dysfunction as reflected by marked elevation of serum creatinine and urea nitrogen $24 \mathrm{~h}$ after IR treatment. Renal function was relatively aggravated in bpv-treated mice, whose serum creatinine and urea nitrogen were much higher than vehicle-treated mice (Fig. 1B, 1C). The results indicated that AKI was successfully induced in our IR model.

\section{Inhibition of PTEN activity aggravates renal fibrosis}

A large amount of evidence indicates that the development of renal fibrosis follows an episode of IR injury [20-22]. To assess the effect of PTEN activity on IR-induced kidney damage, kidney sections were stained with PAS and scored for histological injury 14 days after IR treatment. On a semi-quantitative scale, which includes glomerulosclerosis, interstitial disease, fibrosis and vascular injury, mice undergoing sham treatment had minimal kidney damage, and IR induction caused a great increase in the severity of kidney injury, which was substantially exacerbated in mice after bpv treatment (Fig. 2A and Table 1). Sirius red staining showed that IR-treated mice developed significant collagen deposition in the kidneys compared with sham-treated mice at 14 days after IR. These fibrotic responses were significantly increased in bpv-treated mice (Fig. 2B and 2C).

Table 1. Inhibition of PTEN activity aggravates IR-induced Kidney Injury, ${ }^{* * *} \mathrm{p}<0.001$ vs. Vehicle Sham; $+++p<0.001$ vs. bpv IR; \#\#p<0.01 vs. Vehicle IR, $n=6$ each

\begin{tabular}{lcccc}
\hline Experimental Group & Glomerular Injury & Tubulointerstitial Disease & Vascular Damage & Total Injury \\
\hline Vehicle-Sham & $0.19 \pm 0.02$ & $0.18 \pm 0.02$ & $0.16 \pm 0.03$ & $0.52 \pm 0.05$ \\
Vehicle-IR & $2.73 \pm 0.39^{* * *}$ & $4.35 \pm 0.54^{* * *}$ & $1.39 \pm 0.19^{* * *}$ & $6.03 \pm 0.69^{* * *}$ \\
bpv-Sham & $0.21 \pm 0.03^{+++}$ & $0.23 \pm 0.02^{+++}$ & $0.17 \pm 0.03^{+++}$ & $0.51 \pm 0.04^{+++}$ \\
bpv-IR & $3.89 \pm 0.42^{\# \#}$ & $6.12 \pm 0.46^{\# \#}$ & $3.88 \pm 0.34^{\# \#}$ & $8.64 \pm 0.76^{\# \#}$ \\
\hline
\end{tabular}

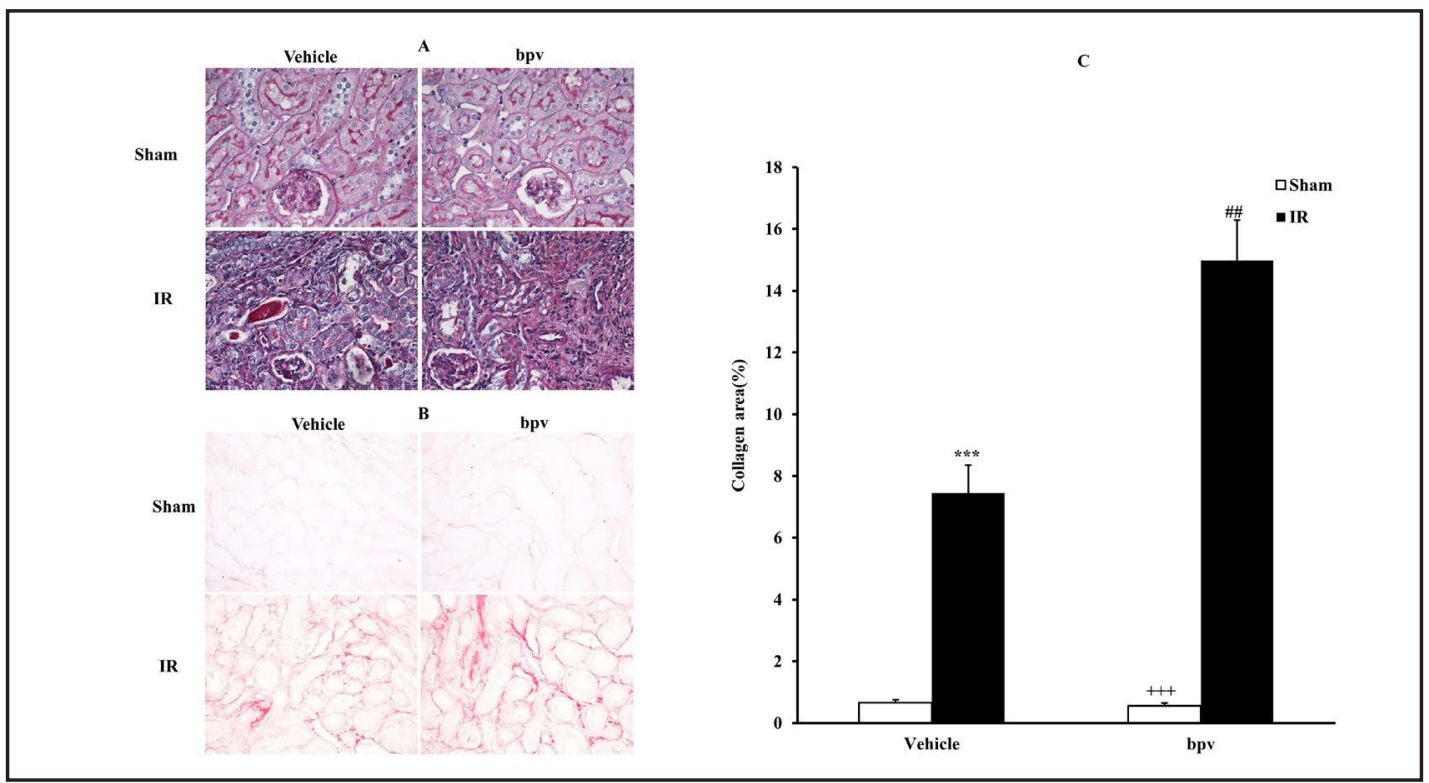

Fig. 2. Inhibition of PTEN activity exacerbated renal injury and fibrosisA. Representative photomicrographs of PAS-stained sections showing kidney damage in vehicle and bpv mice 14 days after IR. Scale bar, 50 $\mu \mathrm{m}$. B. Representative photomicrographs of kidney sections from vehicle and bpv mice 14 days after IR or sham treatment stained with Sirius red for assessment of total collagen deposition. Scale bar, $50 \mu \mathrm{m}$. C. Quantitative analysis of interstitial collagen content in the kidneys of vehicle and bpv mice. ${ }^{* * *} \mathrm{p}<0.001$ vs. Vehicle Sham; ${ }^{++} \mathrm{p}<0.001$ vs. bpv IR; ${ }^{\# \#} \mathrm{p}<0.01$ vs. Vehicle IR, $\mathrm{n}=6$ each. 


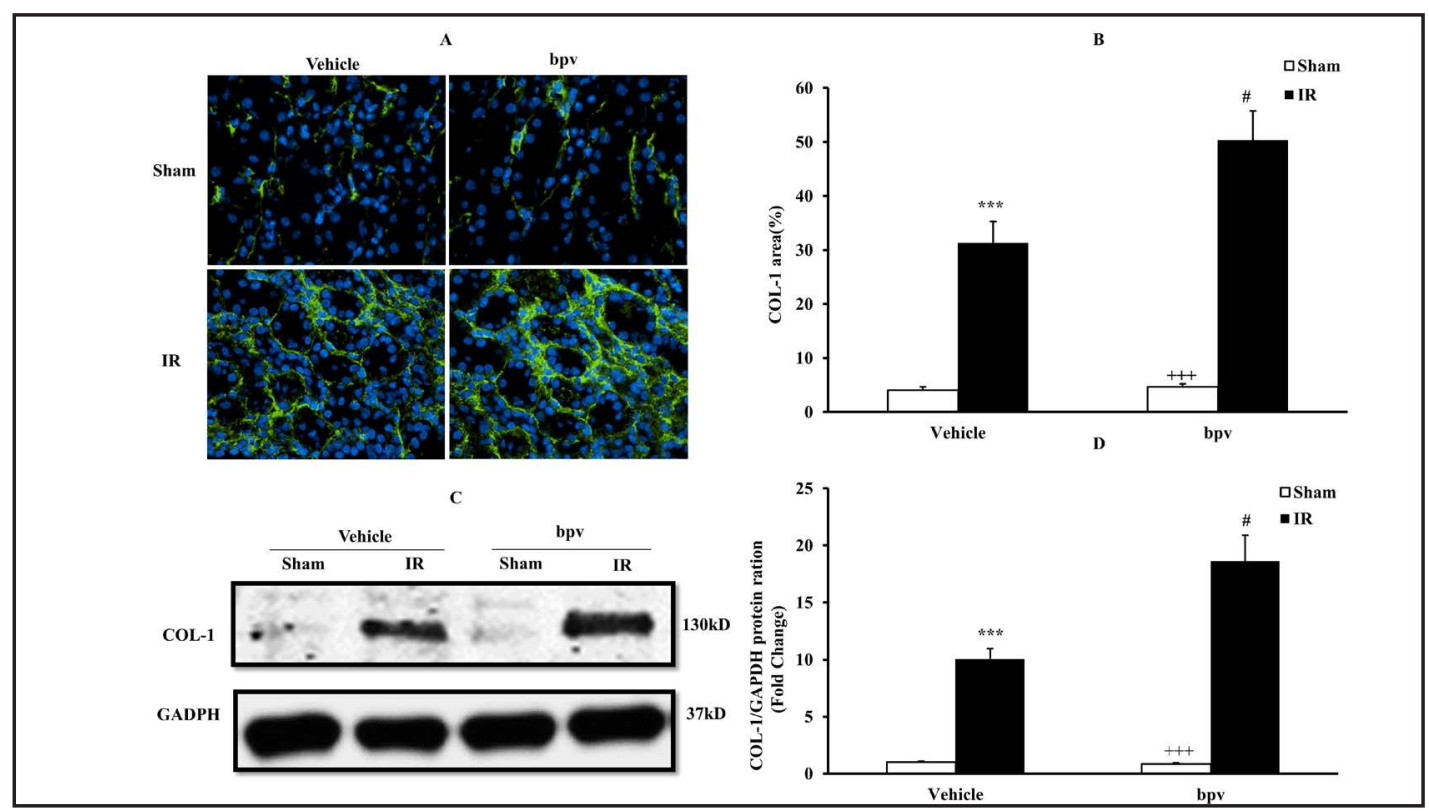

Fig. 3. Inhibition of PTEN activity increased collagen I expressionA. Representative photomicrographs of collagen I immunofluorescence staining in kidneys of vehicle and bpv mice 14 days after IR or sham treatment. Scale bar, $50 \mu \mathrm{m}$. B. Quantitative analysis of collagen I positive areas in the kidneys of vehicle and bpv mice. ${ }^{* * *} \mathrm{p}<0.001$ vs. Vehicle Sham; ${ }^{++} \mathrm{p}<0.001$ vs. bpv IR; ${ }^{*} \mathrm{p}<0.05$ vs. Vehicle IR, $\mathrm{n}=6$ each. C. Representative Western blots show protein levels of collagen I in the kidneys of vehicle and bpv mice 14 days after IR or sham treatment. D. Quantitative analysis of collagen I protein expression in the kidneys of vehicle and bpv mice. ${ }^{* * *} \mathrm{p}<0.001$ vs. Vehicle Sham; ${ }^{+++} \mathrm{p}<0.001$ vs. bpv IR; ${ }^{\#} \mathrm{p}<0.05$ vs. Vehicle IR, $\mathrm{n}=6$ each.

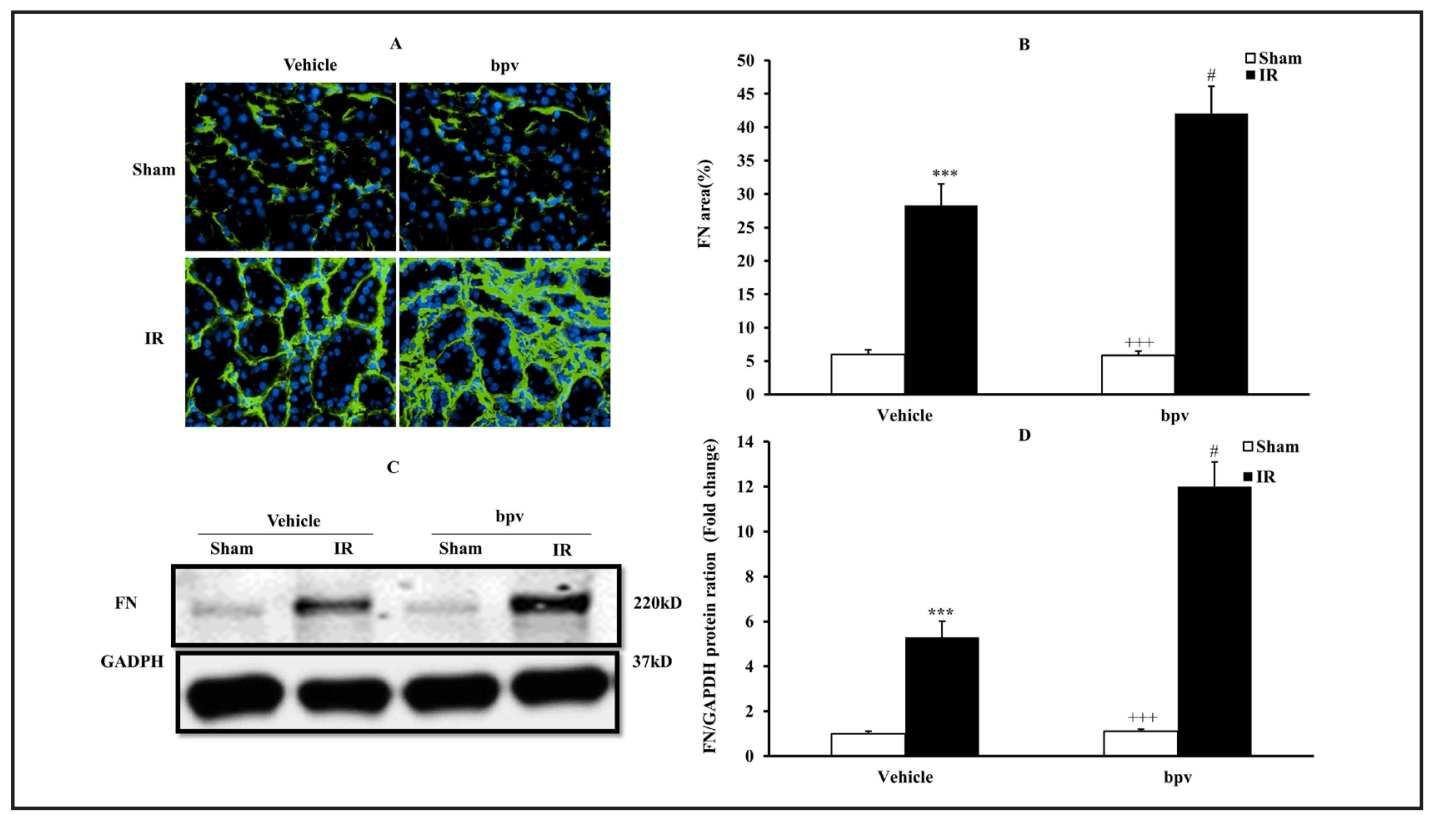

Fig. 4. Inhibition of PTEN activity increased fibronectin expressionA. Representative photomicrographs of fibronectin immunofluorescence staining in kidneys of vehicle and bpv mice 14 days after IR or sham treatment. Scale bar, $50 \mu \mathrm{m}$. B. Quantitative analysis of fibronectin positive areas in the kidneys of vehicle and bpv mice. ${ }^{* * *} \mathrm{p}<0.001$ vs. Vehicle Sham; ${ }^{++} \mathrm{p}<0.001$ vs. bpv IR; ${ }^{\#} \mathrm{p}<0.05$ vs. Vehicle IR, $\mathrm{n}=6$ each. C. Representative Western blots show protein levels of fibronectin in the kidneys of vehicle and bpv mice 14 days after IR or sham treatment. D. Quantitative analysis of fibronectin protein expression in the kidneys of vehicle and bpv mice. ${ }^{* * *} \mathrm{p}<0.001$ vs. Vehicle Sham; ${ }^{+++} \mathrm{p}<0.001$ vs. bpv IR; ${ }^{\#} \mathrm{p}<0.05$ vs. Vehicle IR, $\mathrm{n}=6$ each. 
To evaluate the effect of PTEN activity on ECM protein expression, immunofluorescence staining was carried out to detect the expression levels of collagen I and fibronectin, which are two major ECM proteins. The protein levels of collagen I (Fig. 3A, 3B) and fibronectin (Fig. 4A, 4B) were significantly increased in the kidneys of IR-treated mice. The protein levels of these ECM proteins significantly increased in the kidneys of bpv-treated mice after IR. Consistent with immunofluorescence staining, Western blot analysis demonstrated that inhibition of PTEN activity up-regulated levels of collagen I (Fig. 3C, 3D) and fibronectin (Fig. 4C, 4D) in the kidneys after IR. These data indicated that the inhibition of PTEN activity contributed significantly to ECM protein expression in the kidneys following IR-induced AKI.

\section{Fibroblast Accumulation}

To determine if inhibition of PTEN activity influenced the myofibroblast population, kidney sections 14 days after IR were stained for $\alpha$-SMA, a marker of myofibroblasts, and examined with a fluorescence microscope. The results revealed that bpv-treated mice exhibited a significant increase in the number of $\alpha-\mathrm{SMA}^{+}$myofibroblasts in the kidneys after IR treatment compared with vehicle mice (Fig. 5A and 5B). Consistent with these findings, Western blot analysis showed that inhibition of PTEN activity significantly increased protein expression levels of $\alpha$-SMA (Fig. 5C and 5D) in the kidneys after IR treatment in bpv mice compared with vehicle mice. In addition, the protein expression of vimentin, another marker of myofibroblasts, was also increased (Fig. 5E and 5F).

Proliferation of interstitial fibroblasts is considered the hallmark of progressive renal fibrosis. Therefore, we detected the expression of ki67 using immunohistochemistry. The results demonstrated that proliferation of interstitial fibroblasts was significantly increased

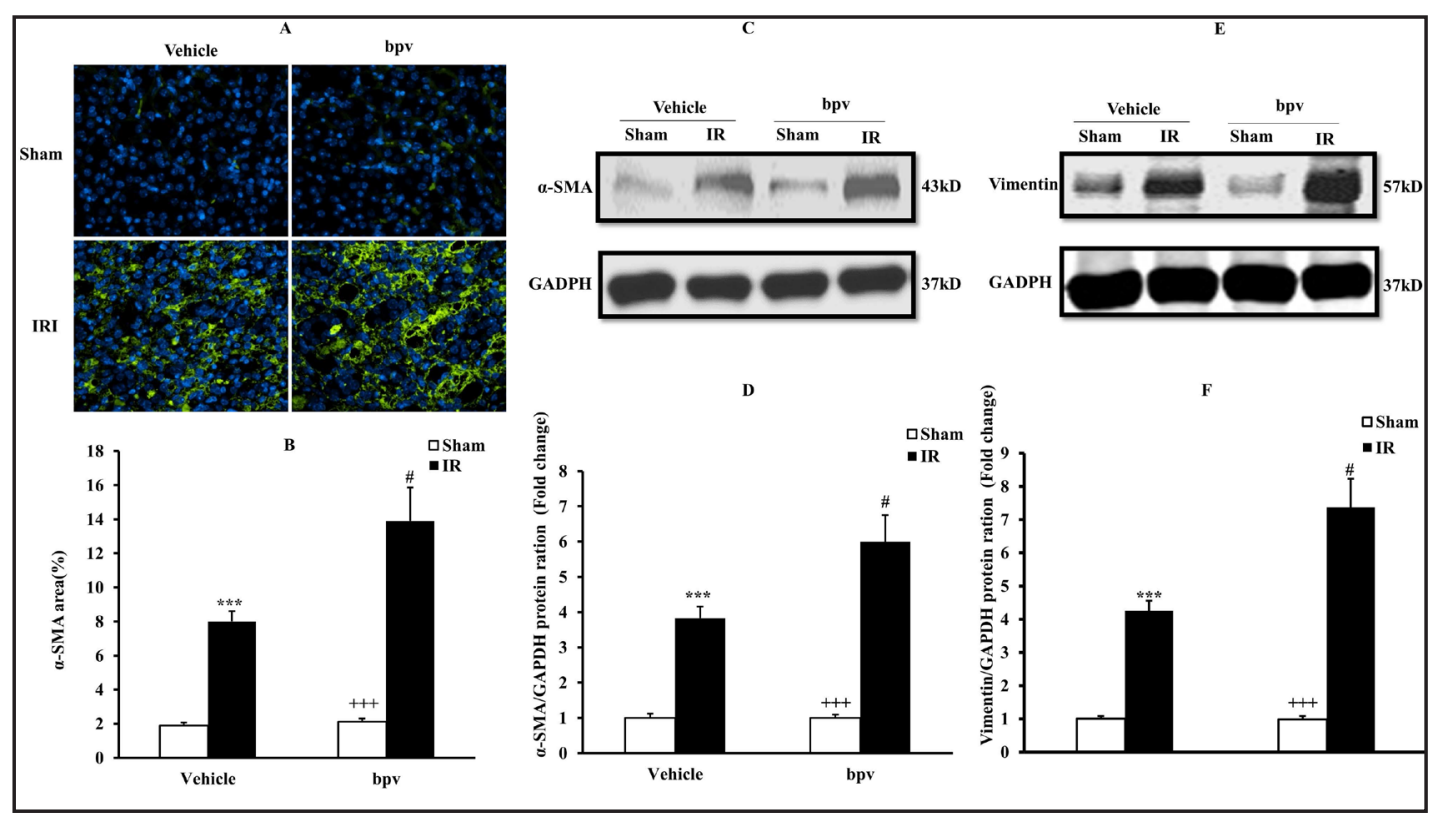

Fig. 5. Inhibition of PTEN activity increased $\alpha$-SMA and vimentin expressionA. Representative photomicrographs of $\alpha$-SMA immunofluorescence staining in the kidneys of vehicle and bpv mice 14 days after IR or sham treatment. Scale bar, $50 \mu \mathrm{m}$. B. Quantitative analysis of $\alpha$-SMA positive area in the kidneys of vehicle and bpv mice. ${ }^{* * *} \mathrm{p}<0.001$ vs. Vehicle Sham; ${ }^{+++} \mathrm{p}<0.001$ vs. bpv IR; ${ }^{\#} \mathrm{p}<0.05$ vs. Vehicle IR, $\mathrm{n}=6$ each. C. Representative Western blots show the protein levels of $\alpha$-SMA in the kidneys of vehicle and bpv mice 14 days after IR or sham treatment. D. Quantitative analysis of $\alpha$-SMA protein expression in the kidneys of vehicle and bpv mice. ${ }^{* * *} \mathrm{p}<0.001$ vs. Vehicle Sham; ${ }^{+++} \mathrm{p}<0.001$ vs. bpv IR; ${ }^{*} \mathrm{p}<0.05$ vs. Vehicle IR, $\mathrm{n}=6$ each. E. Representative Western blots show the protein levels of vimentin in the kidneys of vehicle and bpv mice 14 days after IR or sham treatment. F. Quantitative analysis of vimentin protein expression in the kidneys of vehicle and bpv mice. ${ }^{* * *} \mathrm{p}<0.001$ vs. Vehicle Sham; ${ }^{+++} \mathrm{p}<0.001$ vs. bpv IR; ${ }^{\#} \mathrm{p}<0.05$ vs. Vehicle IR, $\mathrm{n}=6$ each.

\section{KARGER}




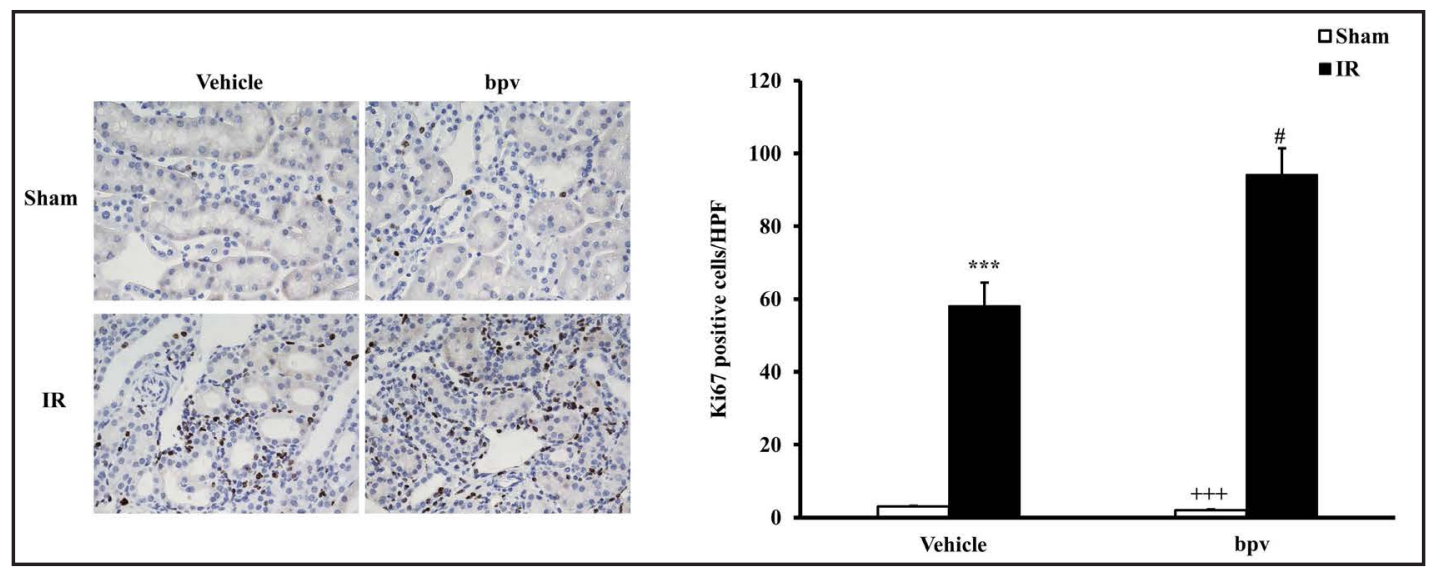

Fig. 6. Inhibition of PTEN activity increased ki67 expression in the kidney after cisplatin treatmentA. Representative photomicrographs of kidney sections stained for ki67 (brown) and counterstained with hematoxylin (blue) in the kidneys of vehicle and bpv mice after cisplatin or saline treatment. B. Quantitative analysis of ki $67^{+}$cells in the kidneys of vehicle and bpv mice after cisplatin or saline treatment. ${ }^{* * *}$ p $<0.001$ vs. Vehicle Sham; ${ }^{++} \mathrm{p}<0.001$ vs. bpv IR; ${ }^{\#} \mathrm{p}<0.05$ vs. Vehicle IR, $\mathrm{n}=6$ each.

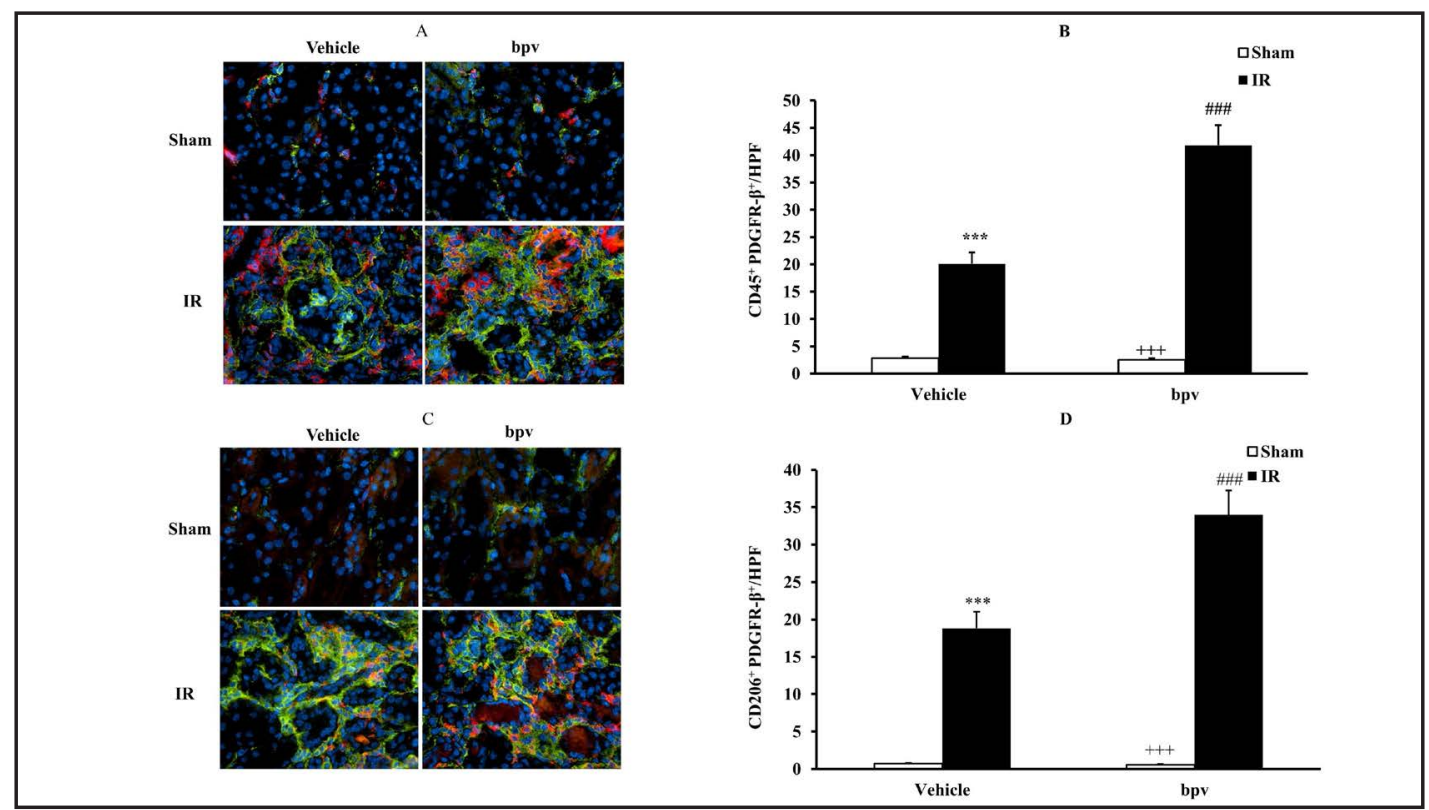

Fig. 7. Inhibition of PTEN activity increased bone marrow-derived fibroblast accumulation and myofibroblast formation in the kidneysA. Representative photomicrographs of kidney sections from vehicle and bpv mice 14 days after IR or sham treatment, stained for CD45 (green), PDGFR- $\beta$ (red), and DAPI (blue). Scale bar, $50 \mu \mathrm{m}$. B. Quantitative analysis of CD $45^{+}$and PDGFR- $\beta^{+}$fibroblasts in the kidneys of vehicle and bpv mice 14 days after IR or sham treatment. C. Representative photomicrographs of kidney sections from vehicle and bpv mice 14 days after IR or sham treatment, stained for CD206 (green), PDGFR- $\beta$ (red), and DAPI (blue). Scale bar, $50 \mu \mathrm{m}$. D. Quantitative analysis of $\mathrm{CD}_{206^{+}}$and PDGFR- $\beta^{+}$fibroblasts in the kidneys of vehicle and bpv mice 14 days after IR or sham treatment. ${ }^{* * *} \mathrm{p}<0.001$ vs. Vehicle Sham; ${ }^{+++} \mathrm{p}<0.001 \mathrm{vs.} \mathrm{bpv}$ IR; \#\# ${ }^{\#<0.001 ~ v s . ~ V e h i c l e ~ I R, ~} n=6$ each.

in the kidneys 14 days after IR, and inhibition of PTEN aggravated the proliferation of interstitial fibroblasts (Fig. 6). Myeloid fibroblasts contribute significantly to the pathogenesis of renal fibrosis [23-26]. Bone marrow-derived fibroblast precursors, termed fibrocytes, are spindle-shaped cells that express hematopoietic markers such as CD45 and CD206, as well as mesenchymal markers such as platelet-derived growth factor receptor- $\beta$ (PDGFRR- $\beta$ ).

\section{KARGER}


To examine the effect of the PTEN activity on the accumulation of myeloid fibroblasts in the kidney, kidney sections at 14 days after IR were double-stained with CD45 and CD206 with PDGFR- $\beta$ and examined with a fluorescence microscope. The results showed that the number of bone marrow-derived fibroblasts that were dual positive for CD45 and PDGFR- $\beta$ (Fig. 7A and 7B) and CD206 and PDGFR- $\beta$ (Fig. 7C and 7D) was significantly increased in the kidneys of IR-treated mice that received bpv i.p. compared with vehicle mice undergoing IR. These data indicated that inhibition of PTEN activity increased the population of fibroblasts in the kidney in response to IR.

\section{Inhibition of PTEN activity increases inflammatory cytokine expression}

To determine the role of PTEN activity on inflammatory cytokine expression in the pathogenesis of IR-induced post renal fibrosis, we examined the effect of bpv on the expression of inflammatory cytokines by real-time PCR at 7 days after IR treatment. The results showed that the mRNA levels of IL- $1 \beta$, IL- 6 , TNF- $\alpha$ and NF- $\kappa B$ in the kidney were increased significantly in the kidneys of vehicle mice after IR treatment compared with sham controls, and the up-regulation of IL- $1 \beta$, IL-6, TNF- $\alpha$ and NF- $\kappa$ B were greatly increased in the kidneys of bpv-treated mice following IR treatment (Fig. 8).

\section{Inhibition of PTEN activity activates PI3K/Akt signaling pathway in IR-induced post renal} fibrosis

The PTEN/PI3K/AKT pathway regulates multiple cellular functions, including cell growth, differentiation, proliferation, survival, motility, invasion and intracellular trafficking $[27,28]$. To explore the mechanisms of inhibition of PTEN activity in the pathogenesis of IR-induced post renal fibrosis, we examined whether bpv affected the protein expression



Fig. 8. Inhibition of PTEN activity increased the gene expression of proinflammatory molecules in the kidneys after cisplatin treatment.A. Quantitative analysis of IL-1 $\beta$ mRNA expression in the kidneys from vehicle and bpv mice at 7 days after sham or IR treatment. ${ }^{* * *} \mathrm{p}<0.001$ vs. Vehicle Sham; ${ }^{++} \mathrm{p}<0.001 \mathrm{vs.} \mathrm{bpv}$ IR; ${ }^{\# \#} \mathrm{p}<0.001$ vs. Vehicle IR, $\mathrm{n}=6$ each. B. Quantitative analysis of IL-6 mRNA expression in kidneys from vehicle and bpv mice 7 days after sham or IR treatment. ${ }^{* * *} \mathrm{p}<0.001 \mathrm{vs.} \mathrm{Vehicle} \mathrm{Sham;}{ }^{++} \mathrm{p}<0.001 \mathrm{vs}$. bpv IR; \#\#\# $\mathrm{p}<0.001$ vs. Vehicle IR, $\mathrm{n}=6$ each. C. Quantitative analysis of TNF- $\alpha$ mRNA expression in kidneys from vehicle and bpv mice 7 days after sham or IR treatment. ${ }^{* * *} \mathrm{p}<0.001$ vs. Vehicle Sham; ${ }^{+++} \mathrm{p}<0.001$ vs. bpv IR; ${ }^{\# \#} \mathrm{p}<0.001$ vs. Vehicle IR, $\mathrm{n}=6$ each. D. Quantitative analysis of NF- $\kappa B$ mRNA expression in kidneys from vehicle and bpv mice 7 days after sham or IR treatment. ${ }^{* * *} \mathrm{p}<0.001$ vs. Vehicle Sham; ${ }^{+++} \mathrm{p}<0.001$ vs. bpv IR; $\# \#$ p $<0.001$ vs. Vehicle IR, $n=6$ each. $n=6$ each. GAPDH, glyceraldehyde-3-phosphate dehydrogenase. 


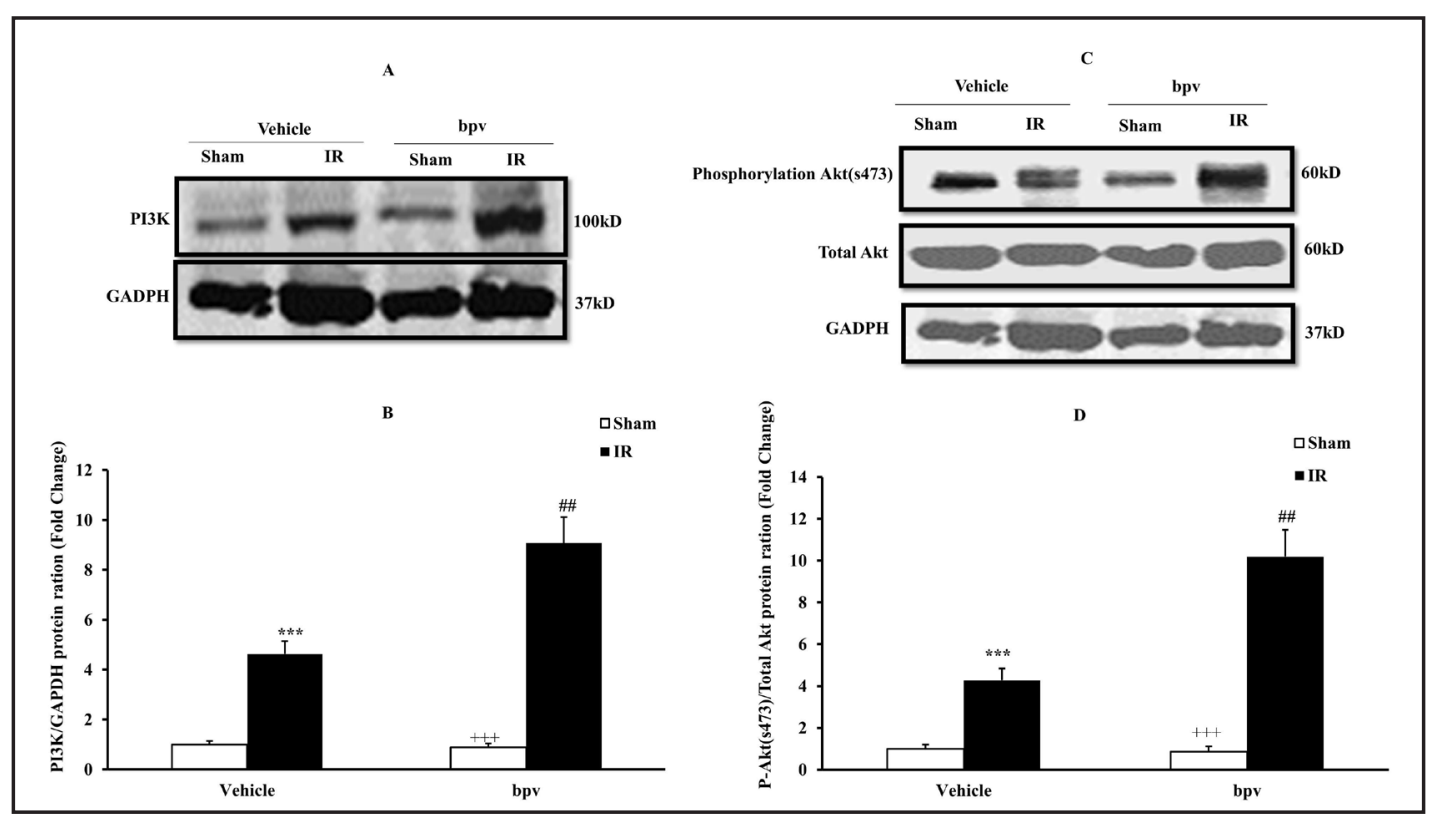

Fig. 9. Inhibition of PTEN activity increased PI3K expression and Akt(s473) phosphorylation in the pathogenesis of IR-induced renal fibrosis.A. Representative Western blots showing PI3K protein in the kidneys from vehicle and bpv mice 7 days after sham or IR treatment. B. Quantitative analysis of PI3K protein in the kidneys from vehicle and bpv mice 7 days after sham or IR treatment. ${ }^{* * *} \mathrm{p}<0.001$ vs. Vehicle Sham; ${ }^{++} \mathrm{p}<0.001$ vs. bpv IR; ${ }^{\# \#} \mathrm{p}<0.01$ vs. Vehicle IR, $\mathrm{n}=6$ each. C. Representative Western blots showing the phosphorylation level of Akt(s473) in the kidneys from vehicle and bpv mice 7 days after sham or IR treatment. D. Quantitative analysis of the phosphorylation level of Akt(s473) in kidneys from vehicle and

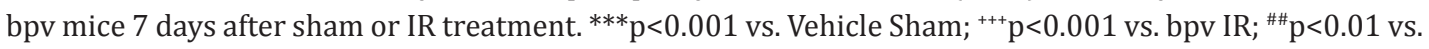
Vehicle IR, $\mathrm{n}=6$ each.

of PI3K and phosphorylation of Akt (s473) at 7 days after IR treatment. Our result showed that IR treatment increased protein expression of PI3K detected by Western blotting in the kidneys of vehicle mice. The protein expression of PI3K was significantly increased in the kidneys of bpv-treated mice after IR treatment compared with vehicle bpv-treated mice (Fig. 9A and 9B). Western blot analysis showed that inhibition of PTEN activity significantly increased the phosphorylation of Akt in the kidneys after IR treatment in bpv-treated mice compared with vehicle mice (Fig. 9C and 9D). These data indicated that PTEN is involved in the pathogenesis of IR-induced post renal fibrosis via the PI3K/Akt signaling pathway.

\section{Discussion}

As a global public health concern, AKI is associated with high morbidity, mortality, and healthcare costs [29]. In recent years, there have been a number of studies about progressive CKD following AKI [30-32]. However, the molecular mechanism of AKI-induced renal fibrosis, which is the main pathological feature of CKD, remains unclear. It was reported that PTEN modulated renal fibrosis by regulating renal extracellular matrix production via signaling pathways such as Akt, SMAD3 and p53(Ser15)[16]. Despite a growing body of research trying to clarify the role of PTEN in the pathogenesis of renal fibrosis induced by kidney injury [15, 33], the potential mechanisms are still unknown. In the present study, we demonstrated that (1)Specific inhibition of PTEN activity exacerbated IR-induced post renal fibrosis; (2) Specific inhibition of PTEN activity further increased the proliferation of interstitial fibroblasts and myeloid fibroblast accumulation in the kidney during the pathogenesis of IR-induced renal fibrosis; (3) Specific inhibition of PTEN activity increased inflammatory cytokines in the 
kidney during IR-induced renal fibrosis; and (4) PTEN activity is involved in IR-induced post renal fibrosis via the PI3K/Akt signaling pathway. These results indicated that PTEN, via the PI3K/Akt signaling pathway, regulated inflammation, myeloid proliferation, and interstitial fibroblast accumulation, playing a critical role in the pathogenesis of IR-induced post renal fibrosis.

Current evidence suggests that PTEN may play an important role in the development of fibrosis in multiple viscera by several mechanisms, including the Akt signaling pathway. Kral JB proved that inhibition of PTEN activity exacerbated BLM-induced lung fibrosis via activation of proinflammatory and profibrotic pathways [34]. Niu et al. provided strong evidence that miR-1273g-3p directly modulated the PTEN signaling pathway in HCV-related liver fibrosis [35]. Previous studies showed a relationship between the PTEN/Akt pathway and heart fibrosis [36]. Some evidence directly illustrated that PTEN regulated renal fibrosis during diabetes mellitus [37]. At present, there are few studies regarding PTEN involvement in AKI-induced renal fibrosis. We first demonstrated that inhibition of PTEN activity aggravated post renal fibrosis after IR-induced AKI.

Because the activation of fibroblasts is regarded as a key event in the pathogenesis of renal fibrosis [38], up-regulation of a-SMA expression is the common proof of renal fibrosis in many studies [12]. Moreover, the expression of vimentin is a feature present in quiescent mesenchymal cells, whereas the expression of functional proteins is a feature of activated fibroblasts [39]. In combination with ki67 staining, which is a classic marker of proliferation, our results showed that inhibition of PTEN activity aggravated the proliferation of interstitial fibroblasts in the kidney in response to IR treatment. Although the origin of the fibroblasts remains controversial, mounting evidence indicates that bone marrow-derived fibroblast precursors contribute substantially to the development of renal interstitial fibrosis [23]. Bone marrow-derived fibroblast precursors are derived from mononuclear cells [40]. CD45 and CD206 are the markers of hematopoietic cells, and PDGFR- $\beta$ is a mesenchymal marker. A previous study showed that bone marrow-derived fibroblasts contributed significantly to the development of renal fibrosis [41]. In the present study, we showed that PTEN activity had a pathologically important effect on recruiting myeloid fibroblasts into the kidney and proliferation of interstitial fibroblasts in the kidney during renal fibrosis. Inhibition of PTEN activity aggravated bone marrow-derived fibroblast accumulation and proliferation of interstitial fibroblasts in the kidney in response to IR treatment.

There is a significant association between inflammatory cytokines and the development of CKD[42]. Proinflammatory cytokines, such as TNF- $\alpha$, IL-6, and IL-1 $\beta$, have been implicated in the pathogenesis of kidney injury induced CKD[43]. A recent experimental study has shown that NF- $\mathrm{kB}$ mediates inflammatory reactions that contribute to renal fibrosis [44]. Moreover, it is not only involved in the proliferation of interstitial fibroblasts in the kidney in response to injury, but there is a strong correlation between inflammation and the proliferation of interstitial fibroblasts or bone marrow-derived fibroblasts in the pathogenesis of renal fibrosis. Studies indicate that inflammatory factor activation and fibroblast proliferation and recruitment are interactive $[45,46]$. Therefore, in the present study, we investigated the effect of PTEN activity on inflammatory cytokines in the kidney during IR-induced renal fibrosis. Our results indicated that inhibition of PTEN activity promoted the recruitment of inflammatory cells in the kidney during IR-induced renal fibrosis.

PTEN resulted in dysregulated activation of the PI3K signaling network, which is recognized as one of the most common driving events in prostate cancer development [47]. Recently, as a new agent, PTEN has been recommended to fight against fibrogenesis. Modulation of PI3K/Akt signaling may promote fibrogenesis in the development of chronic renal disease [48]. Direct evidence indicates that suppression of cytoplasmic PTEN expression and Akt activation upregulate renal fibrosis [49]. However, there is no firm evidence that PTEN activity mediates the PI3K/Akt signaling pathway to modulate IR-induced post renal fibrosis. Our study proved that PTEN alleviated fibrosis in the pathogenesis of AKI-induced renal fibrosis through inhibition of the PI3K/Akt signaling pathway. 
Taken together, our study indicated that PTEN activation plays a pivotal role in alleviating fibrosis in the pathogenesis of IR-induced post renal fibrosis through inhibition of the PI3K/Akt signaling pathway. It furthermore down-regulates bone marrow-derived fibroblast accumulation and the proliferation of interstitial fibroblasts, macrophages and inflammatory molecule production in the kidney. These data suggest that future therapeutic strategies targeting PTEN/PI3K/Akt signaling may be of potential benefit in AKI-induced CKD.

\section{Acknowledgements}

This work was supported by Science and Technology Project of Guangdong Province 2014A020212612 (to Jun Zhou), Natural Science Foundation of Guangdong Province 2016A030313376 (to Jiying Zhong) and General Program of Traditional Chinese Medicine Bureau of Guangdong Province 20171199 (to Youling Fan).

\section{Disclosure Statement}

None of the authors have financial relationships with biotechnology manufacturers, pharmaceutical companies, or other commercial entities with an interest in the subject matter or materials discussed in the manuscripts.

\section{References}

1 Lang J, Zuber K, Davis J: Acute kidney injury. JAAPA 2016;29:51-54.

$\longrightarrow 2$ Ulusoy S, Ar1 D, Ozkan G, Cansız M, Kaynar K: The Frequency and Outcome of Acute Kidney Injury in a Tertiary Hospital: Which Factors Affect Mortality? Artif Organs 2015;39:597-606.

3 Hsu RK, Hsu CY: The Role of Acute Kidney Injury in Chronic Kidney Disease. Semin Nephrol 2016;36:283292.

4 Palant CE, Amdur RL, Chawla LS: The Acute Kidney Injury to Chronic Kidney Disease Transition: A Potential Opportunity to Improve Care in Acute Kidney Injury. Contrib Nephrol 2016;187:55-72.

5 Muñoz-Félix JM, Fuentes-Calvo I, Cuesta C, Eleno N, Crespo P, López-Novoa JM, Martínez-Salgado C: Absence of K-Ras Reduces Proliferation and Migration But Increases Extracellular Matrix Synthesis in Fibroblasts. J Cell Physiol 2016;231:2224-2235.

6 Knafo S, Esteban JA: PTEN: Local and Global Modulation of Neuronal Function in Health and Disease. Trends Neurosci 2017;40:83-91.

7 Wise HM, Hermida MA, Leslie NR: Prostate cancer, PI3K, PTEN and prognosis. Clin Sci (Lond) 2017;131:197-210.

8 Cheng Y, Tian Y, Xia J, Wu X, Yang Y, Li X, Huang C, Meng X, Ma T, Li J: The role of PTEN in regulation of hepatic macrophages activation and function in progression and reversal of liver fibrosis. Toxicol Appl Pharmacol 2017;317:51-62.

-9 Kral JB, Kuttke M, Schrottmaier WC, Birnecker B, Warszawska J, Wernig C, Paar H, Salzmann M, Sahin E, Brunner JS, Österreicher C, Knapp S, Assinger A, Schabbauer G: Sustained PI3K Activation exacerbates BLM-induced Lung Fibrosis via activation of pro-inflammatory and pro-fibrotic pathways. Sci Rep 2016;6:23034.

10 Zhou X, Zang X, Ponnusamy M, Masucci MV, Tolbert E, Gong R, Zhao TC, Liu N, Bayliss G, Dworkin LD, Zhuang S: Enhancer of Zeste Homolog 2 Inhibition Attenuates Renal Fibrosis by Maintaining Smad7 and Phosphatase and Tensin Homolog Expression. J Am Soc Nephrol 2016;27:2092-2108.

11 Sakai N, Wada T: T Helper 2 Cytokine Signaling in Bone Marrow-Derived Fibroblasts: A Target for Renal Fibrosis. J Am Soc Nephrol 2015;26:2896-2898. 


\section{Cellular Physiology Cell Physiol Biochem 2017;43:1841-1854

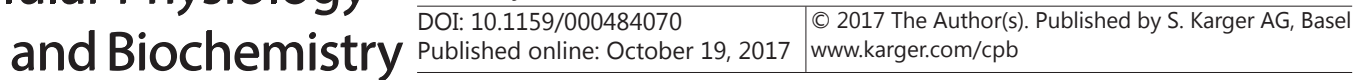

Zhou et al.: Molecular Mechanisms of Post Renal Fibrosis Induced by Acute Kidney Injury (AKI)

12 Yan J, Zhang Z, Yang J, Mitch WE, Wang Y: JAK3/STAT6 Stimulates Bone Marrow-Derived Fibroblast Activation in Renal Fibrosis. J Am Soc Nephrol 2015;26:3060-3071.

13 Kadoya H, Satoh M, Sasaki T, Taniguchi S, Takahashi M, Kashihara N: Excess aldosterone is a critical danger signal for inflammasome activation in the development of renal fibrosis in mice. FASEB J 2015;29:38993910.

14 Dong Y, Yang M, Zhang J, Peng X, Cheng J, Cui T, Du J: Depletion of CD8+ T Cells Exacerbates CD4+ T CellInduced Monocyte-to-Fibroblast Transition in Renal Fibrosis. J Immunol 2016;196:1874-1881.

15 Lan R, Geng H, Polichnowski AJ, Singha PK, Saikumar P, McEwen DG, Griffin KA, Koesters R, Weinberg JM, Bidani AK, Kriz W, Venkatachalam MA: PTEN loss defines a TGF- $\beta$-induced tubule phenotype of failed differentiation and JNK signaling during renal fibrosis. Am J Physiol Renal Physiol 2012;302:F1210-1223.

16 Samarakoon R, Helo S, Dobberfuhl AD, Khakoo NS, Falke L, Overstreet JM, Goldschmeding R, Higgins PJ: Loss of tumour suppressor PTEN expression in renal injury initiates SMAD3- and p53-dependent fibrotic responses. J Pathol 2015;236:421-432.

17 Mavrantoni A, Thallmair V, Leitner MG, Schreiber DN, Oliver D, Halaszovich CR: A method to control phosphoinositides and to analyze PTEN function in living cells using voltage sensitive phosphatases. Front Pharmacol 2015;6:68.

18 Ramesh G, Zhang B, Uematsu S, Akira S, Reeves WB: Endotoxin and cisplatin synergistically induce renal dysfunction and cytokine production in mice. Am J Physiol Renal Physiol 2007;293:F325-332.

19 Xia Y, Entman ML, Wang Y: Critical Role of CXCL16 in Hypertensive Kidney Injury and Fibrosis. Hypertension 2013;62: 1129-1137.

20 Wang M, Weng X, Guo J, Chen Z, Jiang G, Liu X: Metformin alleviated EMT and fibrosis after renal ischemiareperfusion injury in rats. Ren Fail 2016;38:614-621.

21 Han SJ, Kim JH, Kim JI, Park KM: Inhibition of microtubule dynamics impedes repair of kidney ischemia/ reperfusion injury and increases fibrosis. Sci Rep 2016;6:27775.

-22 Masola V, Zaza G, Gambaro G, Onisto M, Bellin G, Vischini G, Khamaysi I, Hassan A, Hamoud S, Nativ O, N Heyman S, Lupo A, Vlodavsky I, Abassi Z: Heparanase: A Potential New Factor Involved in the Renal Epithelial Mesenchymal Transition (EMT) Induced by Ischemia/Reperfusion (I/R) Injury. PLoS One 2016;11:e0160074.

23 Chen G, Lin SC, Chen J, He L, Dong F, Xu J, Han S, Du J, Entman ML, Wang Y: CXCL16 recruits bone marrowderived fibroblast precursors in renal fibrosis. J Am Soc Nephrol 2011;22:1876-1886.

24 Li J, Deane JA, Campanale NV, Bertram JF, Ricardo SD: The contribution of bone marrowderived cells to the development of renal interstitial fibrosis. Stem Cells 2007;25:697-706.

25 Mack M, Yanagita M: Origin of myofibroblasts and cellular events triggering fibrosis. Kidney Int 2015;87:297-307.

26 Grimm PC, Nickerson P, Jeffery J, Savani RC, Gough J, McKenna RM, Stern E, Rush DN: Neointimal and tubulointerstitial infiltration by recipient mesenchymal cells in chronic renalallograft rejection. $\mathrm{N}$ Engl J Med 2001;345:93-97.

-27 Luo F, Shi J, Shi Q Xu X, Xia Y, He X: Mitogen-Activated Protein Kinases and Hypoxic/Ischemic Nephropathy. Cell Physiol Biochem 2016;39:1051-1067.

-28 Malemud CJ: The PI3K/Akt/PTEN/mTOR pathway: a fruitful target for inducing cell death in rheumatoid arthritis? Future Med Chem 2015;7:1137-1147.

29 Zuk A, Bonventre JV: Acute Kidney Injury. Annu Rev Med 2016;67:293-307.

-30 Brandoni A, Torres AM: Altered Renal Expression of Relevant Clinical Drug Transporters in Different Models of Acute Uremia in Rats. Role of Urea Levels. Cell Physiol Biochem 2015;36:907-916.

-31 Parr SK, Siew ED: Delayed Consequences of Acute Kidney Injury. Adv Chronic Kidney Dis 2016;23:186-194.

-32 Tan HL, Yap JQ, Qian Q: Acute Kidney Injury: Tubular Markers and Risk for Chronic Kidney Disease and End-Stage Kidney Failure. Blood Purif 2016;41:144-150.

-33 McClelland AD, Herman-Edelstein M, Komers R, Jha JC, Winbanks CE, Hagiwara S, Gregorevic P, Kantharidis P, Cooper ME: miR-21 promotes renal fibrosis in diabetic nephropathy by targeting PTEN and SMAD7 Clin Sci (Lond) 2015;129:1237-1249.

34 Kral JB, Kuttke M, Schrottmaier WC, Birnecker B, Warszawska J, Wernig C, Paar H, Salzmann M, Sahin E, Brunner JS, Österreicher C, Knapp S, Assinger A, Schabbauer G: Sustained PI3K Activation exacerbates BLM-induced Lung Fibrosis via activation of pro-inflammatory and pro-fibrotic pathways. Sci Rep 2016;6:23034. 
Zhou et al.: Molecular Mechanisms of Post Renal Fibrosis Induced by Acute Kidney Injury (AKI)

-35 Niu X, Fu N, Du J, Wang R, Wang Y, Zhao S, Du H, Wang B, Zhang Y, Sun D, Nan Y: miR-1273g-3p modulates activation and apoptosis of hepatic stellate cells by directly targeting PTEN in HCV-related liver fibrosis. FEBS Lett 2016;590:2709-2724.

-36 Glass C, Singla DK: MicroRNA-1 transfected embryonic stem cells enhance cardiac myocyte differentiation and inhibit apoptosis by modulating the PTEN/Akt pathway in the infarcted heart. Am J Physiol Heart Circ Physiol 2011;301:2038-2049.

37 Zhu L, Zhao S, Liu S, Liu Q, Li F, Hao J: PTEN Regulates Renal Extracellular Matrix Deposit via Increased CTGF in Diabetes Mellitus. J Cell Biochem 2016;117:1187-1198.

-38 Strutz F, Müller GA: Renal fibrosis and the origin of the renal fibroblast. Nephrol Dial Transplant 2006;21:3368-3370.

39 Galichon P, Hertig A: Epithelial to mesenchymal transition as a biomarker in renal fibrosis: are we ready for the bedside? Fibrogenesis Tissue Repair 2011;4:11.

-40 Quan TE, Cowper S, Wu SP, Bockenstedt LK, Bucala R: Circulating fibrocytes: collagen-secreting cells of the peripheral blood. Int J Biochem Cell Biol 2004;36:598-606.

41 Yang J, Chen J, Yan J, Zhang L, Chen G, He L, Wang Y: Effect of interleukin 6 deficiency on renal interstitial fibrosis. PLoS One 2012;7:e52415.

42 Akchurin OM, Kaskel F: Update on inflammation in chronic kidney disease. Blood Purif 2015;39:84-92.

43 Mehta PK, Griendling KK: Angiotensin II cell signaling: physiological and pathological effects in the cardiovascular system. Am J Physiol Cell Physiol 2007;292:C82-97.

-44 Xu ZJ, Shu S, Li ZJ, Liu YM, Zhang RY, Zhang Y: Liuwei Dihuang pill treats diabetic nephropathy in rats by inhibiting of TGF- $\beta /$ SMADS, MAPK, and NF-kB and upregulating expression of cytoglobin in renal tissues. Medicine (Baltimore) 2017;96:e5879.

-45 Maarouf OH, Aravamudhan A, Rangarajan D, Kusaba T, Zhang V, Welborn J, Gauvin D, Hou X, Kramann R, Humphreys BD: Paracrine Wnt1 Drives Interstitial Fibrosis without Inflammation by Tubulointerstitial Cross-Talk. J Am Soc Nephrol 2016;27:781-790.

46 Yan J, Zhang Z, Jia L, Wang Y: Role of Bone Marrow-Derived Fibroblasts in Renal Fibrosis. Front Physiol 2016;7:61.

47 Wise HM, Hermida MA, Leslie NR: Prostate cancer, PI3K, PTEN and prognosis. Clin Sci (Lond) 2017;131:197-210.

48 Du R, Xia L, Ning X, Liu L, Sun W, Huang C, Wang H, Sun S: Hypoxia-induced Bmi1 promotes renal tubular epithelial cell-mesenchymal transition and renal fibrosis via PI3K/Akt signal. Mol Biol Cell 2014;25:26502659.

49 Yao Y, Wei H, Liu L, Liu L, Bai S, Li C, Luo Y, Zeng R, Han M, Ge S, Xu G: Upregulated DJ-1 promotes renal tubular EMT by suppressing cytoplasmic PTEN expression and Akt activation. J Huazhong Univ Sci Technolog Med Sci 2011;31:469-475. 